\title{
Tacticity effects on the barriers to rotation of the ester methyl group in poly (methyl methacrylate): A deuteron magnetic resonance study
}

\author{
P. M. Cereghetti and R. Kind \\ Institute of Quantum Electronics, ETH-Hönggerberg, CH-8093 Zurich, Switzerland \\ J. S. Higgins \\ Department of Chemical Engineering and Chemical Technology, Imperial College, London SW7 2BY, \\ United Kingdom
}

(Received 17 September 2002; accepted 30 July 2004)

\begin{abstract}
In isotactic poly(methyl methacrylate) (PMMA), we investigate the dynamics of the ester methyl groups by means of deuteron magnetic resonance (DMR) in a deuterated sample. We find that the motion of the $\mathrm{CD}_{3}$-group affects the deuteron spin-lattice relaxation as well as the DMR line shape in a characteristic way. Quadrupolar order spin lattice relaxation measurements between $T$ $=291 \mathrm{~K}$ and $T=70 \mathrm{~K}$ reveal a broad temperature dependent probability distribution of autocorrelation times $\tau_{c}$ for the $2 \pi / 3$ reorientation. This broad distribution corresponds to a temperature independent Gaussian distribution of activation energies $\rho\left(E_{a}\right)$ with variance $\sigma_{E_{a}}$ $=13.8 \pm 0.5 \mathrm{meV}(1.33 \mathrm{~kJ} / \mathrm{mol})$. The line shape transition between $T=70 \mathrm{~K}$ and $T=23 \mathrm{~K}$ is explained with the freezing in of the methyl group reorientation. By comparing our results in an $88 \%$ isotactic sample with results obtained from a 50\% syndiotactic, $30 \%$ atactic, and $20 \%$ isotactic sample of a previous investigation, we demonstrate the higher local order of the $88 \%$ isotactic sample, which corresponds to a ratio of 1.6 in the relative width $\sigma_{E_{a}} / E_{a}$ of the $E_{a}$ distribution. We show that different stereospecific forms of PMMA can be easily distinguished by the characteristics of their line shape transition between $T=70 \mathrm{~K}$ and $T=23 \mathrm{~K}$. (C) 2004 American Institute of Physics. [DOI: 10.1063/1.1797173]
\end{abstract}

\section{INTRODUCTION}

Because of their important role in determining physical properties, the relaxation processes in polymeric materials have been studied extensively using a very large variety of spectroscopic, mechanical, and dielectric techniques. ${ }^{1}$ Well below the glass transition temperature $T_{g}$ the $\alpha$ process associated with motion of segments of the main polymer backbone is frozen. As the temperature nears $T_{g}$ these segments begin to move and eventually, well above $T_{g}$, allow the translational motion associated with the liquid state. ${ }^{2-4}$ Polymer molecules with side groups may have a number of other relaxation processes in the glassy state, associated with the reorientation of these side groups. Poly(methyl methacrylate) (PMMA) (see Fig. 1) is particularly rich in this respect and as a consequence has been the subject of extensive studies. The structure of the repeat unit in Fig. 1(a) allows one to identify potential motion of the $\alpha$-methyl and the ester- methyl groups about the $\mathrm{C}-\mathrm{C}$ and $\mathrm{C}-\mathrm{O}$ bonds, respectively, as well as reorientation of the whole ester side group about the $\mathrm{C}-\mathrm{C}$ bond linking it to the backbone (although in principle reorientation about the $\mathrm{C}-\mathrm{O}$ bond of the whole $\mathrm{O}-\mathrm{CH}_{3}$ group is possible, it has not been detected). The rotation of the whole ester group has usually been termed the $\beta$ relaxation, and its motion falls conveniently in the frequency range of NMR techniques. Its molecular nature has been clarified by the use of both $2 d$ and $3 d$ exchange NMR using ${ }^{13} \mathrm{C}$ chemical shifts and ${ }^{2} \mathrm{H}$ quadrupolar anisotropies as probes of dynamical information. ${ }^{5}$ The experiments show that the O-C-O plane of the side group undergoes $180^{\circ}$ flips accompanied by a rearrangement of the main polymer backbone. Any significant motion of the methoxy group about the $\mathrm{C}-\mathrm{OCH}_{3}$ bond was ruled out as mentioned above.

The higher frequency motions of the two methyl groups ( $\delta$ relaxation for the ester $\mathrm{CH}_{3}$ and $\gamma$ for the $\alpha$ ) are conveniently studied by neutron quasielastic and inelastic scattering. The ester relaxation in particular has been the subject of a series of studies from several groups. ${ }^{6-10}$ Neutron scattering differentiates between hydrogen and deuterium so that deuterium labeling is a convenient way of highlighting the motion of the part of the molecule of interest. In fact, the deuterium cross section is much less than that of hydrogen so that the labeling leaves the hydrogenous segments showing and suppresses the signal from those parts containing deuterium. As we shall later discuss neutron and ${ }^{2} \mathrm{H}$ NMR sample requirements are therefore in a way complementary.

The model for the motion of a methyl group about the bond connecting it to the remaining part of a molecule is particularly simple. The symmetry of the group leads to a threefold symmetric potential well whose depth is determined by largely intramolecular factors. The methyl group can exist in any of the three equivalent sites separated by a $2 \pi / 3$ rotation [see Fig. 1(b)]. Within the potential wells six librational states are available - ground state and five excited states. At high temperatures, transitions between these librational levels appear in the far infrared spectrum. At the same time an activated hopping movement is possible over the potential barriers and this appears as a hindered rotational 


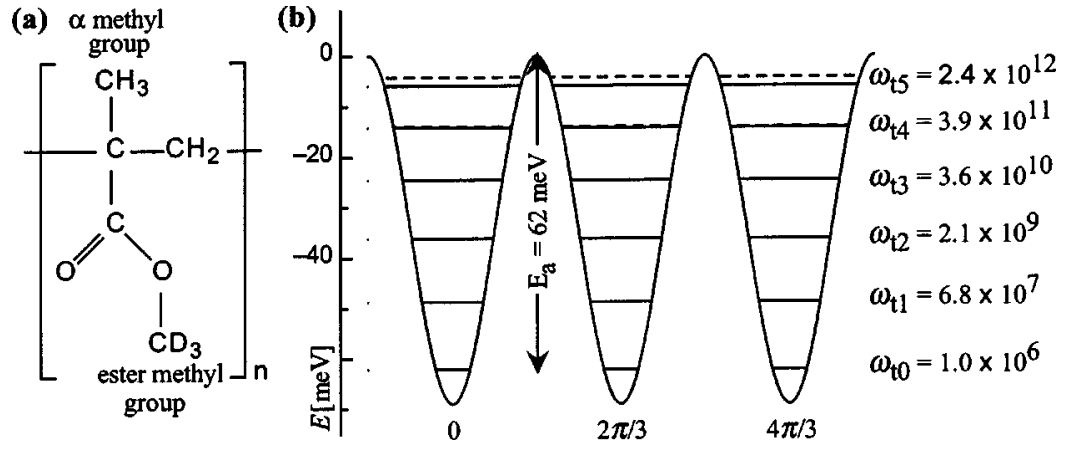

FIG. 1. (a) Chemical structure of the monomer methyl$\alpha_{3}$-2-methylpropenoate as used in this work. (b) Hindering potential ( $V_{3}=69 \mathrm{meV}$ ) of a deuterated methyl group in PMMA. The energy levels of the localized states were obtained from numerical solutions of the corresponding Mathieu equation. The coupling of the wave functions of the single wells splits the librational levels into the sublevels $A$ (solid lines) and $E$ (dashed lines). The corresponding coherent tunnel frequencies $\omega_{t i}(i=0, \ldots, 5)$, in $\mathrm{rad} / \mathrm{s}$, are indicated. motion. The activation energy is largely determined by the difference in energy from the ground state to the top of the barrier.

In earlier papers the quasielastic neutron studies of this hopping motion were confined by the then available resolution to relatively high temperatures and the data appeared consistent with a single relaxation time or a single activation energy. However, as neutron spectrometers with higher resolution became available problems with this interpretation emerged. Effectively, different activation energies were measured, apparently depending on the resolution used. ${ }^{6}$ This situation was eventually clarified by the realization that there is a distribution of activation energies in the system caused either by intramolecular or intermolecular interactions, which vary for each group perhaps due to conformational or packing heterogeneities frozen in at the glass temperature. ${ }^{8,10}$ A further question arose about the apparent persistence to very low temperatures of quite rapid reorientation-where the frequency extrapolated from higher temperatures would fall far below the resolution limit of the spectrometer. To explain this observation it is necessary to look more closely at the motion of the methyl group. The coupling of the librational wave functions in each of the threefold symmetric wells leads to splitting into two sublevels. At very low temperatures $(<1 \mathrm{~K})$ only the ground state is occupied and the rotor oscillates coherently between the three wells with an oscillation frequency corresponding to the energy of splitting of the ground state energy level. This process is known as quantum mechanical tunneling "through the barrier" and is well known for methyl groups in crystalline materials. The form of the spectra were well understood for crystalline materials but no sign of the expected behavior could be detected for glassy polymers such as for the ester group in PMMA. This problem was recently clarified by Moreno et al. ${ }^{10}$ who realized that the effect of the distribution of rotational barriers already mentioned means that the tunneling spectrum is drastically broadened and distorted. These authors were able to show that the low temperature motion was correctly assigned to tunneling through the threefold barrier. In their recent paper, ${ }^{10}$ they demonstrated that the crossover from classical hoping to quantum mechanical tunneling can be roughly estimated to occur at a temperature where the frequency of coherent oscillations-the tunneling energybecomes comparable to the frequency of incoherent hopping-the classical rotational energy. The calculations are made complex by the necessity of accounting for the distribution of barriers to rotation but for PMMA ester group the authors predict and observe measurable quantum mechanical effects below $80 \mathrm{~K}$. In fact even for crystalline systems with well defined potential barriers, a generally accepted model for the cross over to classical hopping is still lacking. The standard approach involves a rigid rotor coupled to a bath of oscillators-so-called phonon assisted tunneling that is noncoherent.

Selective deuteration and the available frequency resolution mean that ${ }^{2} \mathrm{H}$ NMR is also a potentially interesting technique for observing the detailed behavior of methyl groups. ${ }^{11,12}$ Unlike ${ }^{1} \mathrm{H}$ NMR which is dominated by dipolar interactions and sensitive to intermolecular interactions, the quadrupolar interactions responsible for ${ }^{2} \mathrm{H}$ NMR are dominated by single particle interactions and the results are directly comparable to the incoherent neutron scattering results. We report in this paper a study of the selectively deuterated material PMMA-D P $_{3}$ In this case, however, it is the deuterated ester group which is studied, and therefore it is important to note the effect of the doubling of the atomic mass on the spectra. The major effect is through the increased moment of inertia and hence a doubling of the rotational constant $B$. In the classical regime this results in an increase by a factor of 2 of the correlation time of the $\mathrm{CD}_{3}$ group, as reported by Cavagnat et al. ${ }^{13}$ The low temperature effects in crystalline materials have been well documented in the literature and result in a considerable lowering of the tunneling frequency. Moreno et al. ${ }^{9,10}$ suggest that for the ester-methyl group in PMMA a shift down by a factor of 40 is possible and demonstrates that for a sample with $\mathrm{CD}_{3}$ groups no tunneling broadening is observed with the available neutron scattering resolution. However, the simplified approach takes no account of multilevel tunneling (the fact that tunneling can occur in all six of the librational levels) nor of the complications of phonon assisted tunneling. Thus, while the tunneling frequencies are indeed substantially lower for a $\mathrm{CD}_{3}$ group than for a $\mathrm{CH}_{3}$ group, it is by no means clear how much this multilevel leakage competes with classical hopping and thus affects the apparent Arrhenius behavior and the transition to the pure tunneling regime.

The aim of the current study is to explore the details of the rotational barriers for the ester- $\mathrm{CD}_{3}$ in PMMA and how it is affected by the local molecular conformation. To this end we present for the first time results for a predominantly isotactic sample with one previously studied which has about $50 \%$ syndiotactic sequences.

In the next two sections we shall present the theory necessary for the analysis of the methyl deuteron NMR relax- 
ation data of the PMMA polymer. Sections III and IV are devoted to the experimental part of this work. In Sec. V we discuss the experimental results. Finally we compare our PMMA results with the ones in Ref. 14 obtained from the investigation of a syndiotactic PMMA sample.

\section{DEUTERON QUADRUPOLAR RELAXATION IN ROTATING $\mathrm{CD}_{3}$-GROUPS}

For the hindered rotation of $\mathrm{CD}_{3}$ groups in a Zeeman field $\mathbf{B}_{0}$ (that defines the quantization axis) the dominant relaxation mechanism is the fluctuating nuclear quadrupole (NQ) interaction in the coordinate system of the diagonal time averaged Hamiltonian. The deuteron electric field gradient (EFG) tensors are static in the coordinate system of the C-D bonds but become time dependent through the basis transformation $R(t)$ corresponding to the reorientation of the groups. The relaxation is thus of quadrupolar nature. The fluctuating spin Hamiltonian is directly coupled via $R(t)$ to the mechanical reorientation of the $\mathrm{CD}_{3}$ groups. The investigation of the deuteron-spin-lattice relaxation in selectively deuterated PMMA thus provides information on this motion as well as on the corresponding hindering potential.

The advantage of deuteron magnetic resonance (DMR) is the fact that the spin Hamiltonian $(\mathcal{H})$ of a deuteron in a C-D bond is strongly dominated by the electrostatic intrabond interaction, whereas the external charge distribution, as well as the nuclear magnetic dipole-dipole interaction have a marginal influence. Thus, the NQ interaction $\left(\mathcal{H}_{Q}\right)$ of the deuterons in the $\mathrm{CD}_{3}$ groups can be considered to first order as a static local property, which depends neither on the orientation of the C-D bond, nor on the temperature. The orientation dependence of the total spin Hamiltonian becomes visible only in the presence of a Zeeman interaction $\mathcal{H}_{z}$ induced by an external magnetic field because of the different relative orientations of the quantization axes of $\mathcal{H}_{z}$ and $\mathcal{H}_{Q}$. The reorientational motions of the C-D bonds are therefore directly mapped into oscillations or fluctuations of $\mathcal{H}=\mathcal{H}_{z}$ $+\overline{\mathcal{H}}_{Q}(t)$, where the time dependence of $\overline{\mathcal{H}}_{Q}$ is a result of the basis transformation from the C-D bond space to the Zeeman space.

The time dependence of $\mathcal{H}$ affects the spin-lattice relaxation, as well as the DMR-line shape. Let us first consider the DMR-spectrum of a single C-D bond which forms an angle $\beta$ with the $\mathbf{B}_{0}$ field. The electrical field gradient tensor at the deuteron site is symmetric with its principal $z$ axis in the bond direction. From first-order perturbation theory the transitions between the states $m=0 \leftrightarrow m= \pm 1$ (Refs. 15-17) give in the high field case $\left(\mathcal{H}_{z} \gg \overline{\mathcal{H}}_{Q}\right)$ the two line frequency spectrum with frequencies

$$
\nu=\nu_{L} \pm \frac{1}{2} \nu_{Q}\left(3 \cos ^{2} \beta-1\right),
$$

where $\nu_{L}$ is the Larmor frequency and $\nu_{Q}$ a fraction of the nuclear quadrupole coupling constant $C_{q}\left(\nu_{Q}=3 C_{q} / 4\right)$. For a static isotropic probability distribution of $\mathrm{C}-\mathrm{D}$ bond directions the superposition of all lines yields the well-known Pake pattern with singularities at $\pm \nu_{Q} / 2$. This corresponds to the low temperature spectrum where the hindered rotation is slow or frozen in.

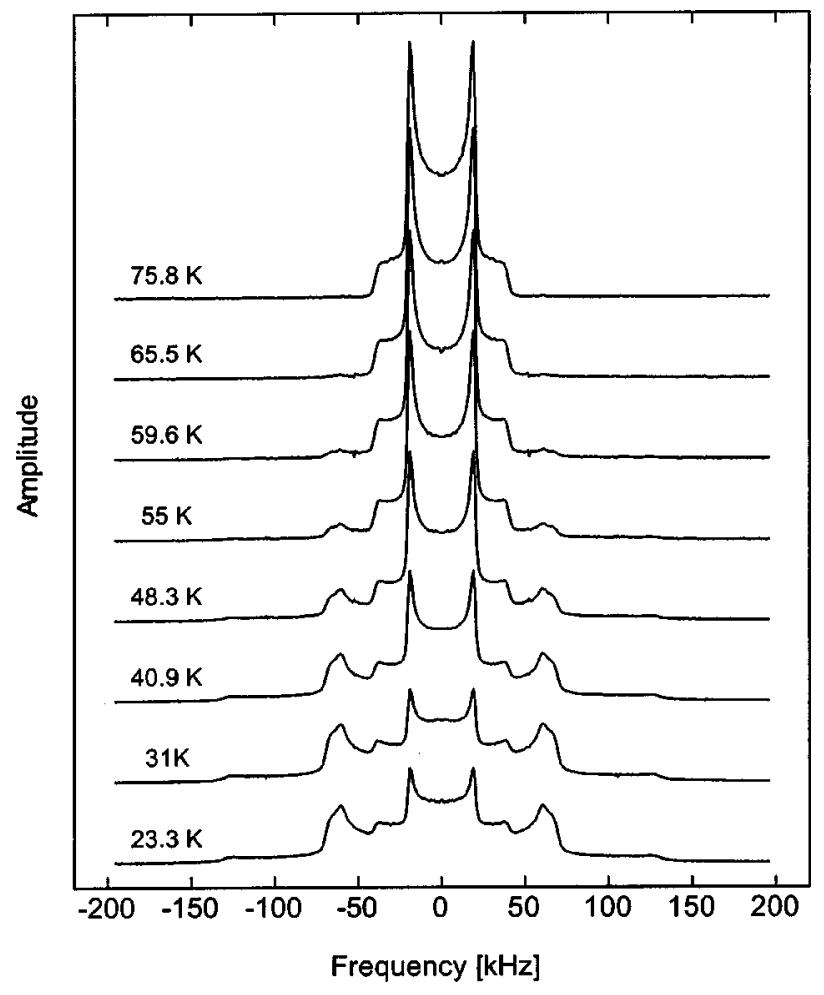

FIG. 2. Experimental DMR line shape transition of the ester methyl deuterons in isotactic PMMA.

A static $\mathrm{CD}_{3}$ group with an arbitrary orientation of its $\mathrm{C}_{3}$ axis with respect to the external magnetic field $\mathbf{B}_{0}$ gives rise to three pairs of DMR lines whose frequency splitting depends essentially on the relative orientation of the C-D bonds and the external magnetic field $\mathbf{B}_{0}$. For very fast reorientation the time dependent terms of the three Hamiltonians are averaged to zero and the static terms become equal, i.e., we are left with a single pair of DMR lines. This is, however, only the case when each deuteron visits the three potential wells for the same amount of time. The corresponding axially symmetric EFG tensor has its principal $z$ axis parallel to the $\mathrm{C}_{3}$ axis and $C_{Q}\left(C_{Q}=152 \mathrm{kHz}\right.$ in PMMA $)$ is reduced by a factor of 3.0 because of the tetrahedral coordination of the $\mathrm{OCD}_{3}$-groups.

The line shape transition from two to six lines and vice versa takes place when the autocorrelation time $\tau_{c}^{H}$ of the reorientation is of the order of the inverse splitting of the three lines of the $m=-1 \leftrightarrow 0$ or $m=0 \leftrightarrow+1$ transitions, respectively. Because of the isotropic distribution of the $\mathrm{C}_{3}$ axes in PMMA the DMR line shape is a Pake pattern for both cases. The fast and the slow motion Pake patterns differ in width by a factor of 3.0. In Fig. 2 such a superposition of Pake patterns corresponding to the fast and slow exchange regimes is shown for the isotactic PMMA stereoisomer. Here fast and slow refer to the quadrupolar splitting.

The formulas for describing nuclear spin-lattice relaxation are in most cases derived from second-order time dependent perturbation theory. It predicts that the relaxation rate between two energy levels is given by the Fourier component (at the transition frequency) of the fluctuation autocorrelation function of the matrix elements relating the two 
levels. A new review focused on NMR in supercooled liquids and glassy solids shows the treatment in detail and presents also a wide bibliography on the topic. ${ }^{18}$ In a spin-lattice experiment one measures either the recovery $M_{z}(t)$ of Zeeman $\operatorname{order}\left(T_{1 Z}\right)$ or the decay $M_{Q}(t)$ of quadrupolar order $\left(T_{1 Q}\right)$, where $M_{Q}(t)$ corresponds to the $M_{x}$ obtained from the transformation of the remaining quadrupolar order. Measuring $T_{1 Q}$ has for a spin $I=1$ in crystalline solids the advantage that $M_{Q}(t)$ is usually monoexponential, whereas $M_{z}(t)$ is biexponential because in addition to the $\Delta m= \pm 1$ transitions $\Delta m= \pm 2$ transitions also contribute to the relaxation. Furthermore, the deuteron $M_{z}(t)$ can be affected by the so called $T_{2}$ hole, which is not the case for $M_{Q}(t) .{ }^{14,19} \mathrm{We}$, therefore, restrict ourselves to $T_{1 Q}$ which for the $2 \pi / 3$ reorientations of the $\mathrm{CD}_{3}$ groups is given by

$$
\frac{1}{T_{1 Q}}=\frac{3}{4}\left(2 \pi C_{Q}\right)^{2} A_{1}\left(\alpha_{0}, \beta\right) \frac{\tau_{c}}{1+\omega_{L}^{2} \tau_{c}^{2}},
$$

where $A_{1}\left(\alpha_{0}, \beta\right)$ is a correction factor for the individual tilt $\beta$ of the $C_{3}$ axis with respect to the external magnetic field $\mathbf{B}_{0}$ and the individual orientation $\left(\alpha_{0}, \alpha_{0}+2 \pi / 3, \alpha_{0}\right.$ $-2 \pi / 3)$ of the three potential wells around the $C_{3}$ axis. $A_{1}\left(\alpha_{0}, \beta\right)$ ranges from 0.06 to 0.47 . This means, for PMMA, that the isotropic probability distribution of the $\mathrm{CD}_{3}$-axis orientation and the constant probability distribution of $\alpha_{0}$ already yield a nonexponential $M_{Q}(t)$. The autocorrelation time $\tau_{c}$ of the $\mathrm{CD}_{3}$ reorientation is expected to follow an Arrhenius law $\left(\tau_{c}=\tau_{\infty} \exp \left[E_{a} / k_{B} T\right]\right)$ with a barrier height $E_{a}$. For stochastic $2 \pi / 3$ reorientation $\tau_{c}$ is related to the mean waiting time $t_{w}$ between two jumps by $\tau_{c}=2 t_{w} / 3$. Equation (2) is valid for an exponential decay of the autocorrelation function. This is the case for uncorrelated $2 \pi / 3$ jumps of the $\mathrm{CD}_{3}$ ensemble and since strongly correlated motions are usually absent below $T_{g}$ we can use Eq. (2) without modifications. However, as we shall see later on, there are other mechanisms that can destroy its validity.

Since the main purpose of this contribution is to obtain information on the barrier height and its expected probability distribution in the glass state of PMMA, which affects only $\tau_{c}$ in Eq. (2), the probability distribution of $A_{1}\left(\alpha_{0}, \beta\right)$ leads to an undesirable convolution of the two distributions that is difficult to disentangle. To eliminate this problem one can measure $M_{Q}(t)$ in frequency space, i.e., frequency resolved and thus via Eq. (1) $\beta$ selective. For $\beta=0^{\circ}$ and $\beta=90^{\circ}$ $A_{1}\left(\alpha_{0}, \beta\right)$ does not depend on the angle $\alpha_{0}$, i.e., $A_{1}\left(\alpha_{0}, 0\right)$ $=2 / 9$ and $A_{1}\left(\alpha_{0}, 0\right)=1 / 3$, respectively. The two angles correspond to the frequencies $\nu_{L} \pm \nu_{Q}$ and $\nu_{L} \pm \nu_{Q} / 2$, respectively. However, both frequencies have a drawback: For $\beta=0$ the intensity is not sufficient for the measurements and for $\beta=90^{\circ}$ the intensity contains a contribution of $14 \%$ of the mirrored spectrum with $\beta=35.27^{\circ}$ according to the \pm sign in Eq. (1). The superposition ratio is not infinite because of the finite homogeneous line width that softens the divergence at $\beta=90^{\circ}$. For $\beta=35.27^{\circ}$ we have $0.1<A_{1}<0.3$, which means that overall we have a sufficiently exponential decay $M_{Q}(t)$. Instead of $\beta=0$ one can choose $\beta=13^{\circ}$ (corresponding to $\nu_{L} \pm 0.92 \nu_{Q}$ ) where the intensity is sufficient and the $\alpha_{0}$ de- pendence still marginal $\left[A_{1}\left(\alpha_{0}, 13^{\circ}\right) \approx 2 / 9\right]$. The problem of the $A_{1}\left(\alpha_{0}, \beta\right)$ distribution can thus be bypassed in this way.

It should be noted that the calculation of $A_{1}\left(\alpha_{0}, \beta\right)$ is based on two assumptions from which the real system might slightly deviate: The C-D bonds are tilted from the $C_{3}$ axis by the exact tetrahedral angle, and the deuteron EFG tensors of the frozen-in system are symmetric in the calculation. This leaves some uncertainty in the value of $A_{1}$, which should, however, not exceed the range $\pm 10 \%$. Calculations revealed that the influence of the asymmetry parameter is rather marginal, whereas a change of the tetrahedral angles affects the ratio of $A_{1}\left(\alpha_{0}, \beta=90^{\circ}\right) / A_{1}\left(\alpha_{0}, \beta=0\right)$ much more strongly than $A_{1}\left(\alpha_{0}, \beta=90^{\circ}\right)$ itself. However, the observed ratio was found to be close to the theoretical value of 1.5 within the experimental error.

\section{EXPERIMENTAL DETERMINATION OF THE AUTOCORRELATION TIME DISTRIBUTION}

The synthesis of the PMMA sample with selective deuteration of the ester methyl group was performed as in Ref. 6 , and an isotacticity of $88 \%$ was obtained. The polydispersity was 2.7 . We hot pressed the sample into a cylindrical shape with the dimensions $\phi=3 \mathrm{~mm}$ and $l=15 \mathrm{~mm}$. The NMR coil was directly wrapped on the sample and placed in a continuous gas flow cryostat with a temperature stability of $\pm 0.05 \mathrm{~K}$. The DMR measurements were performed with a home built NMR spectrometer with a $\mathbf{B}_{0}$ field of $7 \mathrm{~T}$.

The RF pulse length for a $90^{\circ}$ rotation of the deuteron magnetization was $2.3 \mu \mathrm{s}$. This is sufficiently short to obtain an undistorted spectrum of Zeeman order from a $90_{x}-\tau$ $-90_{y}$ echo sequence, even for the case of the broad low temperature Pake pattern. However, the excitation of quadrupolar (zero quantum) order is more selective (i.e., phase distortions in the wings), so that a broad band Jeener-Brockaert sequence $^{20}$ had to be used for a uniform excitation of the entire spectrum. Using an appropriate phase cycling ${ }^{21}$ and a $90^{\circ}$ refocusing readout pulse yields a perfect mapping of quadrupolar order in the Fourier transform of the echo decay. The signal after each pulse sequence was accumulated 12 times. In the frequency space this decay of quadrupolar order as a function of the time span $\tau_{d}$ between excitation and readout was recorded in a $\beta$-selective way as presented in the preceding section.

Figure 3 shows the signal amplitude for $\beta=90^{\circ}(-)$, where the sign indicates the sign in Eq. (1). It is plotted as a function of the delay time $\tau_{d}$ at various temperatures. The signal decays differ clearly from exponential decays and thus according to Sec. II we deal with a distribution of autocorrelation times $\tau_{c}$, i.e., in case of a thermally activated motion, with a probability distribution of activation energies $E_{a}$.

The simplest and most widely used model function is a Gaussian distribution of activation energies $E_{a}$ which leads, via the Arrhenius law, to a log-Gaussian distribution of correlation times

$$
\rho\left(\ln \tau_{c}\right)=\frac{1}{\sqrt{2 \pi} \sigma_{\ln \tau_{c}}} \exp \left[-\frac{1}{2}\left(\frac{\ln \tau_{c}-\overline{\ln \tau_{c}}}{\sigma_{\ln \tau_{c}}}\right)^{2}\right] .
$$




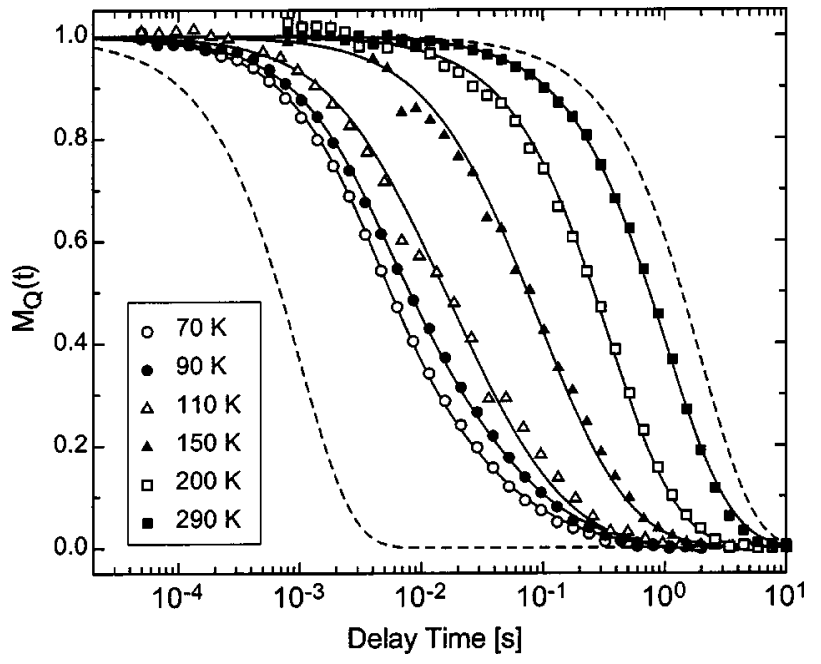

FIG. 3. Nonexponential decays of the quadrupolar order (open circles) as a function of the time for the orientation $\beta=90^{\circ}(-)$ and various temperatures. The solid lines are a fit of Eq. (4) to the data, while the dashed lines are simple exponential decays, shown for comparison.

The corresponding signal decay $Q(t)$ is then a superposition of weighted exponentials

$$
M_{Q}(t, \beta)=M_{Q}(0, \beta) \int_{0}^{\infty} e^{-t / T_{1}\left(\tau_{c}\right)} \rho\left(\ln \tau_{c}\right) d\left(\ln \tau_{c}\right)
$$

where $1 / T_{1 Q}\left(\tau_{c}\right)$ is given by Eq. (2). The solid lines in Fig. 3 are a fit of Eq. (4) to the data points, with $\overline{\ln \tau_{c}}, \sigma_{\ln \tau_{c}}$, and $A_{1}$ as fit parameters. The average $T_{1 Q}$ can be obtained by fitting a single exponential to the data points, which for proper weighting should be taken in equidistant $\ln (t)$ intervals as shown in Fig. 3.

It is well known that for $\tau_{c} \ll 1 / \omega_{L}$ Eq. (2) allows only determination of the product of the mean square fluctuations with $\tau_{c}$, i.e., for a known $C_{Q}$ only the product $A_{1} \tau_{c}$ can be obtained. Only when the $T_{1}$ minimum can be observed in the experiment which occurs at $\tau_{c}=1 / \omega_{L}$ (where the relaxation is independent of $\tau_{c}$ ), can $A_{1}$ be determined separately. Of course one could use the calculated value of $A_{1}$ and fit only $\tau_{c}$, but one has then to accept that the uncertainty of $A_{1}$ is directly transformed into an uncertainty of $\tau_{c}$. For a broad probability distribution of $\tau_{c}$ one expects to obtain for the average $T_{1 Q}$ a broad flat minimum instead of a rather sharp one. Part of such a minimum is indeed observed as shown in Fig. 4, where $\left\langle T_{1 Q}\right\rangle$ is plotted vs $1 / T$, which is (via the Arrhenius law) proportional to $\overline{\ln \tau_{c}}$. Provided that this change of slope is really the result of the $\tau_{c}$ distribution with its upper wing reaching $1 / \omega_{L}, A_{1}$ can be used as independent fit parameter in the temperature region below $110 \mathrm{~K}$.

The mean value $\overline{\ln \tau_{c}}$ and the standard deviation $\sigma_{\ln \tau_{c}}$, which completely characterize the log-Gaussian distribution of correlation times in Eq. (3), are the relevant temperaturedependent fit parameters, whereas $A_{1}$ can be considered as temperature independent because of the stability of the methyl group. Four sets of $M_{Q}(t, \beta)$ data were selected from one set of spectra $\left[\beta=90^{\circ}( \pm)\right.$ and $\left.\beta=13^{\circ}( \pm)\right]$ for each temperature. By a simultaneous least squares fit of Eqs. (2)-(4) to all measured $M_{Q}(t, \beta)$ belonging to the same set and us-

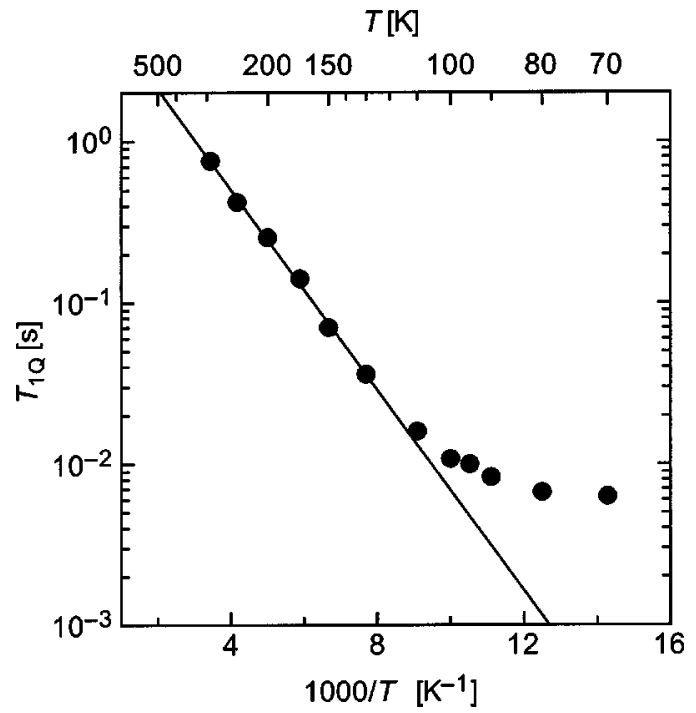

FIG. 4. Average quadrupolar order spin-lattice relaxation time $T_{1 Q}$ vs $1 / T$ for the particular orientation of $\beta=90^{\circ}(-)$. The straight line is a fit to the data points above $125 \mathrm{~K}$. The slope corresponds to an activation energy of $E_{a}=62 \pm 1 \mathrm{meV}$.

ing one single $A_{1}$ for all temperatures and individual couples of $\left(\overline{\ln \tau_{c}}, \sigma_{\ln \tau_{c}}\right)$ for each temperature as fit parameters, we obtained $A_{1}=0.45$, which is well above the uncertainty range mentioned in Sec. II. These values are, however, not very reliable, since the leveling off of $T_{1 Q}$ in Fig. 4 does not only reflect the classical $T_{1}$ minimum, as we shall see later on. We therefore preferred fitting the data with a fixed value of $A_{1}=0.33$ instead. These fitted mean $\tau_{c}$ values are shown in Fig. 5.

The $\log$ and 1/T scaling of the axes in Fig. 5 yields for an Arrhenius behavior a straight line with slope proportional to an activation energy $E_{a}$, which can be associated with the thermal activation energy of the $2 \pi / 3$ methyl reorientation. For temperatures above $125 \mathrm{~K}$ the data points indeed follow a straight line with a slope corresponding to $E_{a}=62 \mathrm{meV}$ $(5.98 \mathrm{~kJ} / \mathrm{mol})$, a value compatible with this kind of motion and with the results for the line-shape transition that we will present in the following section. However, at lower temperatures, starting below $125 \mathrm{~K}$, the apparent values of $\overline{\ln \tau_{c}}$ are leveling off and are smaller than predicted by the Arrhenius law. Possible reasons for this effect will be discussed in Sec. V.

In the temperature region above $125 \mathrm{~K}$ one can apply another fit procedure that takes the Arrhenius law into account by assuming a temperature independent Gaussian probability distribution of $E_{a}$ with standard deviation $\sigma_{E_{a}}{ }^{22}$ For this purpose Eq. (4) has to be modified,

$$
\begin{aligned}
& M_{Q}(t)=M_{Q}(0) \int_{0}^{\infty} \exp \left[-t / T_{1 Q}\left(\tau_{c}\right)\right] \rho\left(E_{a}\right) d E_{a}, \\
& \tau_{c}\left(E_{a}\right)=\tau_{\infty} \exp \left(E_{a} / k_{B} T\right), \\
& \rho\left(E_{a}\right)=\frac{1}{\sqrt{2 \pi} \sigma_{E_{a}}} \exp \left(-\frac{1}{2}\left[\frac{E_{a}-\left\langle E_{a}\right\rangle}{\sigma_{E_{a}}}\right]^{2}\right),
\end{aligned}
$$




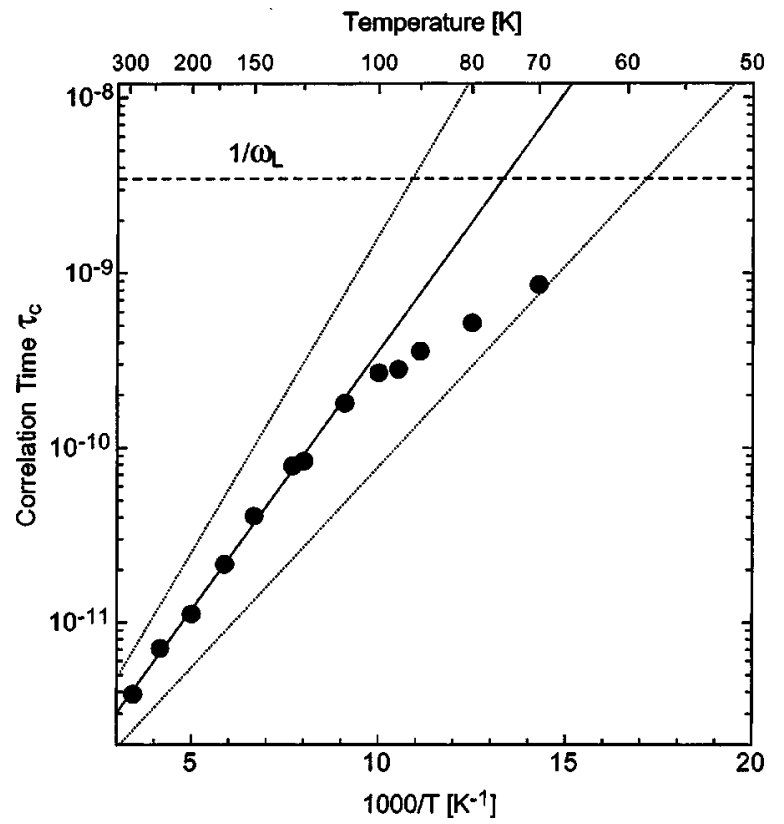

FIG. 5. Mean correlation times $\left(\overline{\tau_{c}}\right)_{i}(i=1, \ldots, 13)$ vs inverse temperature $T$ as obtained from the fit of Eq. (4) to the measured $M_{Q}(t)$. The three straight lines indicate the Arhhenius behavior of the mean values, and the \pm standard deviations of the log-Gaussian $\tau_{c}$-distribution. Below $100 \mathrm{~K}$ the data points deviate for unknown reasons systematically from the Arrhenius behavior. It might be that the conditions for the validity of Eq. (2) are not fulfilled anymore below this temperature.

where $T_{1 Q}\left(\tau_{c}\right)$ is given by Eq. (2). In the corresponding fit procedure $\left\langle E_{a}\right\rangle, \sigma_{E_{a}}$ and the product $A_{1} \tau_{\infty}$ are independent fit parameters. In order to obtain $\tau_{\infty}$ we have to choose a value for $A_{1}$. If all $\overline{\ln \tau_{c}}$ values in Fig. 5 followed the Arrhenius law the choice would just be the fitted value $A_{1}=0.45$ because then the leveling off of $T_{1 Q}$ in Fig. 4 could be assigned entirely to a distribution of the $T_{1}$ minimum. Since, however, the leveling off of $\overline{\ln \tau_{c}}$ also has the same effect on $T_{1 Q}$ the fitted value of $A_{1}$ becomes rather doubtful. It is thus safer to use the theoretical value $A_{1}=1 / 3$ instead. This yields $\left\langle E_{a}\right\rangle=62 \pm 1 \mathrm{meV}(5.98 \mathrm{~kJ} / \mathrm{mol}), \sigma_{E_{a}}=13.3 \pm 0.5 \mathrm{meV}(1.28$ $\mathrm{kJ} / \mathrm{mol})$, and $\tau_{\infty}=(3.9 \pm 0.4) \times 10^{-13} \mathrm{~s}$.

\section{ANALYSIS OF THE DMR LINE SHAPE TRANSITION}

The DMR-line shape transition due to the slowing down of the $\mathrm{CD}_{3} 2 \pi / 3$ reorientation becomes visible in the spectrum below $70 \mathrm{~K}$. In contrast to the transition in single crystals, where the slow and the fast reorientation regimes are separated by a narrow temperature region with almost vanishing signal, in PMMA the two regimes overlap. One observes both the narrow and the broad Pake patterns in the same spectrum. This is a result of the broad probability distribution of $\tau_{c}$ containing three different dynamic states of $\mathrm{CD}_{3}$ groups, the fast and slow reorienting ones as well as those in the transition region. The $\tau_{c}$ distribution was assumed broad enough to neglect the latter. The resulting twostate model for the line shape analysis was first used in Ref. 22.

In the two-state model a superposition of the fractions $F$ and $(1-F)$ of the two normalized Pake patterns, for fast and

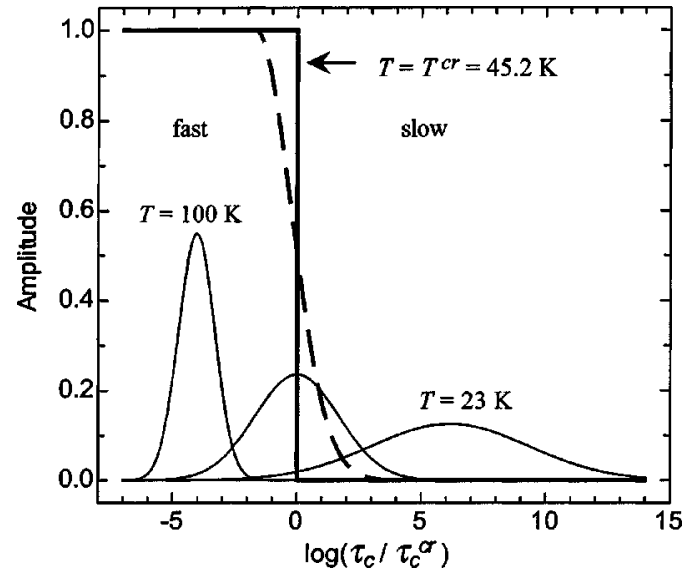

FIG. 6. Illustration of the two-state model: The log-Gaussian distribution with temperature dependent variance of correlation times $\tau_{c}$ is shifted through a step function with step located at $T^{c r}$. The dashed line corresponds to the smoothed step function resulting from taking the transition region into account.

slow reorientation, respectively, are fitted to the normalized measured DMR spectra for various temperatures. The resulting temperature dependent fraction $F(T)$ of fast exchanging $\mathrm{CD}_{3}$ groups is then analyzed by shifting, e.g., a Gaussian probability distribution from left to right through a barrier (step function) and assigning the part left of the barrier as fast and the other part as slow, respectively; see Fig. 6. As mentioned above the transition region is here neglected completely and the question arises, whether this procedure is indeed justified. To answer this question we need deeper insight into the DMR line shape transition in PMMA and have to calculate all spectra and their relative importance in the transition regime.

The theory of the dynamic line shape transition for a set of exchanging lines has been known since the early days of NMR and is treated in many textbooks, see, e.g., A. Abragam (1960). ${ }^{16}$ For the case of a frozen-in deuterated methyl group there are three pairs of NMR transition frequencies centered around the Larmor frequency $\mu_{L}$. The line splitting of each pair is defined by Eq. (1) with three different tilt angles $\left(\beta_{1}, \beta_{2}, \beta_{3}\right)$ of the three $\mathrm{C}-\mathrm{D}$ bonds with respect to the external magnetic field $\mathbf{B}_{0}$. Both signs in Eq. (1) define a trio of transition frequencies $\left(\nu_{1}, \nu_{2}, \nu_{3}\right)$ that depend only on the orientation of the methyl group. From straightforward transformation of the three deuteron EFG tensors from the C-Dcoordinate, via the $\mathrm{C}_{3}$-coordinate, to the $\mathbf{B}_{0}$-coordinate system one can see that the set of three transition frequencies is defined by the angles $\alpha_{0}$ and $\beta$ defined in Sec. II, under the assumption of tetrahedral angles for the $\mathrm{CD}_{3}$ group and symmetry related symmetric EFG tensors for the three deuteron sites. For a fast exchange the three EFG tensors are averaged to a single one with its principal $z$ axis parallel to the $\mathrm{C}_{3}$ axis, i.e., with tilt angle $\beta$ and a single transition frequency $\bar{\nu}$, which is given by $\bar{\nu}=\left(\nu_{1}+\nu_{2}+\nu_{3}\right) / 3$. We therefore have to calculate the line shape transition for three frequencies. According to Ref. 16 the intensity $I(\nu)$ is given by

$$
I(\nu)=\operatorname{Re}\left(\mathbf{p} \cdot \mathbf{A}^{-1} \cdot \mathbf{1}\right)
$$

with $\mathbf{p}=(1 / 3,1 / 3,1 / 3)$ and 


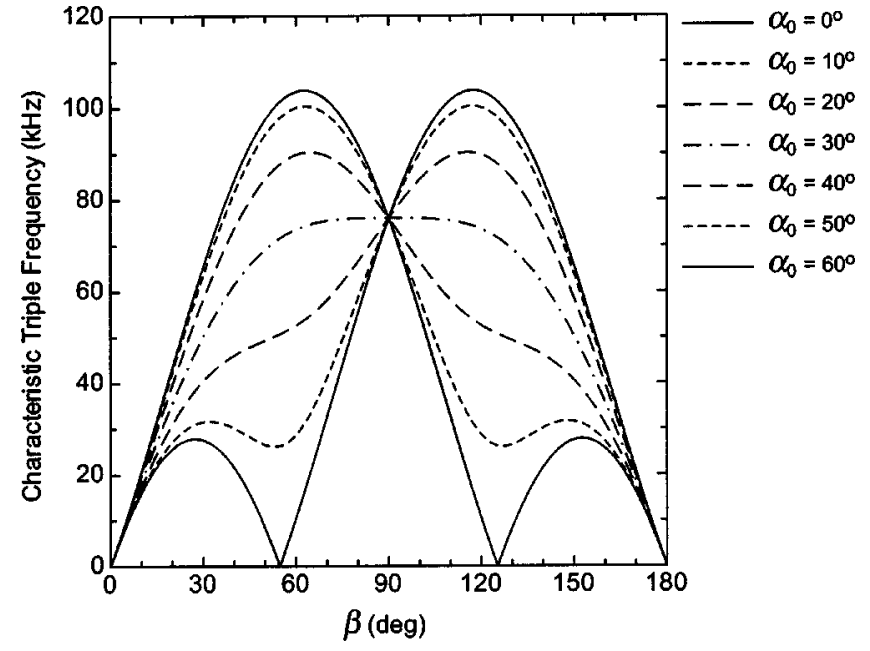

FIG. 7. Characteristic frequency $r$ of the set of DMR lines of a $\mathrm{CD}_{3}$ group as a function of its orientation $\left(\alpha_{0}, \beta\right)$ with respect to the external magnetic field $\mathbf{B}_{0}$. For the calculation of $r$ the value $C_{Q}=152 \mathrm{kHz}$ determined for the ester methyl deuterons in PMMA was used.

$$
\begin{aligned}
A= & 2 \pi i\left(\begin{array}{ccc}
\nu_{1}-\nu & 0 & 0 \\
0 & \nu_{2}-\nu & 0 \\
0 & 0 & \nu_{3}-\nu
\end{array}\right) \\
& +\left(\begin{array}{ccc}
-2 k & k & k \\
k & -2 k & k \\
k & k & -2 k
\end{array}\right) .
\end{aligned}
$$

Here $k$ denotes the transition rate between the three orientations. According to Sec. II the mean life time $t_{w}$ of one orientation is $3 \tau_{c} / 2$ and the probability of making either a $+2 \pi / 3$ or a $-2 \pi / 3$ jump is 0.5 , which yields $k=1 /\left(3 \tau_{c}\right)$. In PMMA we have at low temperatures a huge variety of different DMR frequency sets and to make any predictions on the line shape transition, we need to characterize them.

Any set of three real numbers can be thought of as solutions of a third-order equation $\left(x-x_{1}\right)\left(x-x_{2}\right)\left(x-x_{3}\right)$ $=0, \quad$ i.e., $\quad x^{3}-\left(x_{1}+x_{2}+x_{3}\right) x^{2}+\left(x_{1} x_{2}+x_{1} x_{3}+x_{2} x_{3}\right) x$ $-x_{1} x_{2} x_{3}=0$. For $x_{i}=\nu_{i}-\bar{\nu}$ the second-order term vanishes and the solutions are given by $x_{i}=r \cos \left(\phi+\alpha_{i}\right)$ with $\alpha_{i}=0$, $+2 \pi / 3,-2 \pi / 3$. The autocorrelation time $\tau_{c}^{t r}$ that defines the center of the triple to single line transition is proportional to $r^{-1}$, but does not depend on the angle $\phi$, nor on the average frequency $\bar{\nu}$. For $r$ we get

$$
r=2 \sqrt{-\left(x_{1} x_{2}+x_{1} x_{3}+x_{2} x_{3}\right) / 3} .
$$

The characteristic frequency $r$ for $C_{Q}=152 \mathrm{kHz}$ depends on the angles $\alpha_{0}$ and $\beta$ as shown in Fig. 7. The probability distribution function $G(r)$ was calculated for an isotropic distribution of $C_{3}$ axes and the first moment $M_{1}[G(r)]$ $=\langle r\rangle=63.3 \mathrm{kHz}$ corresponds to the average value of $r$. Tests of Eq. (6) as a function of $\tau_{c}$ revealed that the center of the transition is at $\tau_{c}^{t r}=(2 \pi r)^{-1}$. We therefore define a reference value $\tau_{r e f}=(2 \pi\langle r\rangle)^{-1}$ for further calculations. The total spectrum consists of a superposition of all individual trio spectra in the system for a given $\tau_{c}$. To avoid ambiguities in

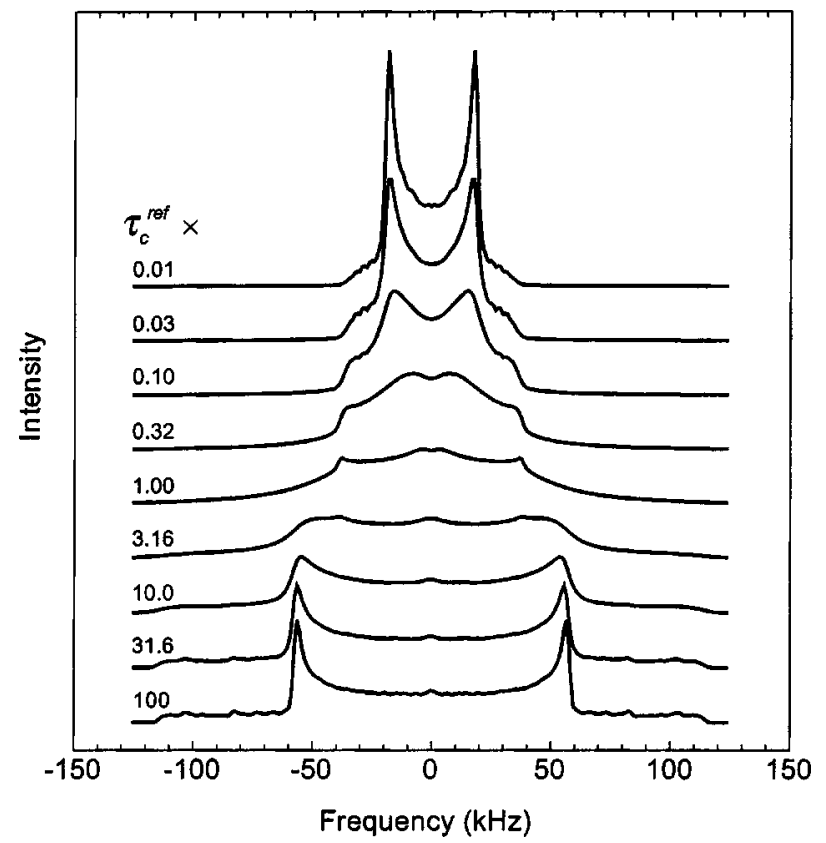

FIG. 8. Calculated monodispersive DMR line shape transition for an isotropic distribution of $C_{3}$ axes of the reorienting $\mathrm{CD}_{3}$ groups with $C_{Q}$ $=152 \mathrm{kHz}$.

calculating the angle $\beta(\Delta \nu)$ from the inversion of Eq. (1) only the plus sign of Eq. (1) was used yielding for fast exchange the half Pake spectrum

$$
\rho_{\text {fast }}(\Delta \nu)=\left(3+18 \frac{\Delta \nu}{\nu_{Q}}\right)^{-2}, \quad-\frac{\nu_{Q}}{6} \leqslant \Delta \nu \leqslant+\frac{\nu_{Q}}{3},
$$

where $\Delta \nu=0$ corresponds to the Larmor frequency $\nu_{L}$. The distribution $\rho_{\text {fast }}(\Delta \nu)$ was used to weight the calculated spectra at $\beta(\Delta \nu)$. An interesting but unimportant detail is that the corresponding slow exchange half spectrum is defined by the minus sign in Eq. (1), i.e.,

$$
\rho_{\text {slow }}(\Delta \nu)=\left(-3+6 \frac{\Delta \nu}{\nu_{Q}}\right)^{-2}, \quad-\frac{\nu_{Q}}{6} \leqslant \Delta \nu \leqslant+\frac{\nu_{Q}}{2} \text {. }
$$

The calculated monodisperse full spectra for the transition region with $\tau_{c}$ as parameter are shown in Fig. 8. This clearly demonstrates that the transition region extends over four orders of magnitude and can thus by no means be neglected.

This is a consequence of the distribution of transition frequencies $r$ according to Fig. 7, i.e., even for a single correlation time we have fast and slow exchanging $\mathrm{CD}_{3}$ groups. To retain some of the striking simplicity of the two-state model we have applied the two-state fit described above to the spectra of Fig. 8. Instead of the parameter $F(T)$ we use now the parameter $\psi\left[{ }^{10} \log \left(\tau_{c}\right)\right]$. (The idea behind this is that, by applying this fit to the polydisperse experimental spectra, we do essentially the same to each of the monodisperse subspectra according to the $\tau_{c}$ distribution of the real system.) The resulting fractions $\psi\left[{ }^{10} \log \left(\tau_{c}\right)\right]$ of fast exchanging $\mathrm{CD}_{3}$ groups are on a smooth curve which can described within $1 \%$ error by a half Gaussian with amplitude 1.0 and variance 2.068 in ${ }^{10} \log \left(\tau_{c}\right)$ coordinates, see dashed line in Fig. 6. An 


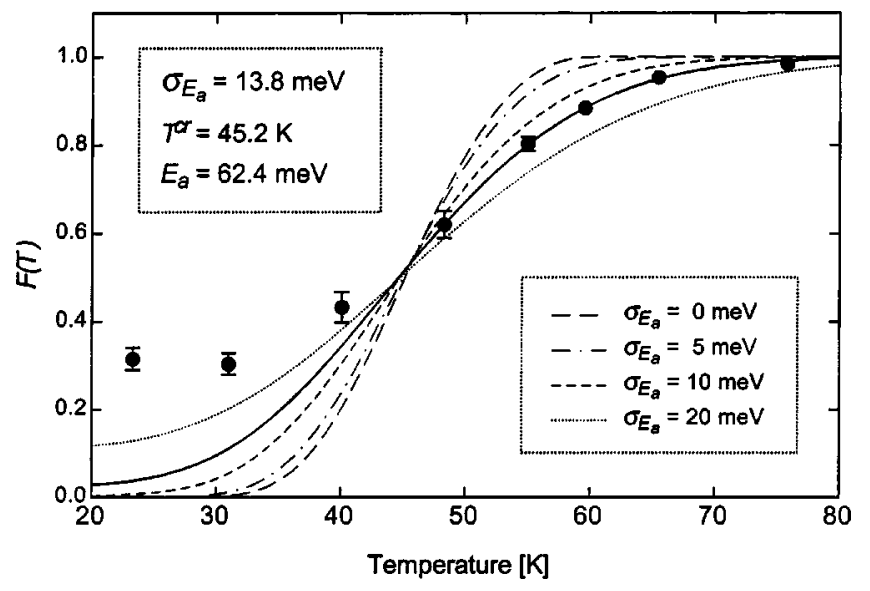

FIG. 9. Fraction of fast exchanging $\mathrm{CD}_{3}$ groups vs temperature. The $F(T)$ data are resulting from a two-state fit of the experimental DMR spectra shown in Fig. 2. The solid line is a fit of the improved two-state model to the data points above $45 \mathrm{~K}$. Additional curves are displayed to give an impression of the sensitivity of the method with respect to $\sigma_{E}$. The data points below $45 \mathrm{~K}$ deviate systematically from the Arrhenius behavior, which can be assigned to the onset of phonon assisted tunneling.

important point, that we call pivot, is defined by $\psi\left[{ }^{10} \log \left(\tau_{c}\right)\right]=0.50$. The corresponding critical correlation time becomes $\tau_{c}^{c r}=0.549 \tau_{r e f} \approx\left(2 \pi \nu_{Q}\right)^{-1}$. The quadrupolar coupling constant $C_{Q}$ can serve thus as a clock to measure $\tau_{c}$.

The unknown parameters involved in the line shape transition are $E_{a}, \sigma_{E_{a}}$, and $\tau_{\infty}$. In view of the special situation it is more convenient to replace $\tau_{\infty}$ by the experimental pivot temperature $T^{c r}$ as fit parameter: $\ln \tau_{\infty}=\ln \tau_{c}^{c r}-E_{a} / k_{B} T^{c r}$. The aim is to obtain a formula for the fraction $F(T)$ of fast exchanging $\mathrm{CD}_{3}$ groups. For the step function shown in Fig. 6 an analytical expression can be given for $F(T)$,

$$
F(T)=\frac{1}{2}\left[\operatorname{erf}\left(\frac{E_{a}\left(T / T^{c r}-1\right)}{\sqrt{2} \sigma_{E_{a}}}\right)+1\right],
$$

where $\operatorname{erf}(x)$ is the error function. Note that this function is in $E_{a}$ coordinates and that it does not contain the quadrupolar coupling constant. The only link to the ${ }^{10} \log \left(\tau_{c}\right)$ coordinate system of Fig. 6 is $T^{c r}$. Furthermore, it contains only two independent fit parameters: $T^{c r}$ and the ratio $E_{a} / \sigma_{E_{a}}$. Thus, e.g., $E_{a}$ has to be taken from the $T_{1 Q}$ experiment. A fit of Eq. (10) to the data points above $45 \mathrm{~K}$ in Fig. 9 yields $T^{c r}$ $=(44.4 \pm 0.3) \mathrm{K}$ and $E_{a} / \sigma_{E_{a}}=3.5 \pm 0.1$.

For the transition function $\psi\left[{ }^{10} \log \left(\tau_{c}\right)\right]$, however, no analytical formula for $F(T)$ can be given. It is not a simple convolution of the two Gaussians since $\sigma_{E_{a}}$ and $\sigma\left\{\psi\left[{ }^{10} \log \left(\tau_{c}\right)\right]\right\}$ are constant in different coordinate systems. Nevertheless $F(T)$ can be calculated numerically to be fitted to the experiment. Though $E_{a}$ and $\sigma_{E_{a}}$ become less dependent on each other, it is not sufficient for a separate determination, so that also here $E_{a}$ has to be taken from $T_{1 Q}$ measurements. The result including the fit parameters is shown in Fig. 9 where the data points were obtained from a two-state fit of the measured DMR spectra shown in Fig. 2. Note that in Fig. 9 only the data points above $45 \mathrm{~K}$ could be used for the fit, since the ones below this temperature show a significant leveling off which will be discussed below.

The fitted parameters are $T^{c r}=45.2 \pm 0.2 \mathrm{~K}$ and $E_{a} / \sigma_{E_{a}}$ $=4.5 \pm 0.1$. I It should be noted that this result is only valid for a Gaussian distribution of activation energies. Asymmetric distributions can lead to considerable shifts of $T^{c r}$ and $E_{a} / \sigma_{E_{a}}$.

Using the mean activation energy determined from $T_{1 Q}$ $\left(\left\langle E_{a}\right\rangle=62 \pm 1 \mathrm{meV}, \quad 5.98 \mathrm{~kJ} / \mathrm{mol}\right)$, we get $\sigma_{E_{a}}=13.8$ $\pm 0.5 \mathrm{meV}(1.33 \mathrm{~kJ} / \mathrm{mol})$. The comparison with the results of the original two-state model shows that the correction in $E_{a} / \sigma_{E_{a}}$ is about $+28 \%$. It is therefore advisable to use the more accurate model presented here, which takes the line shape transition region into account.

The three lowest data points in Fig. 9 deviate significantly from the solid line. In fact the fraction of the fast reorienting $\mathrm{CD}_{3}$ groups is much too high for classical thermally activated hopping. The reason for this is very likely phonon-assisted tunneling through the barriers of the threefold methyl group potential. ${ }^{23-28}$

As mentioned in the Introduction section, such tunneling has been observed in the neutron scattering data from $\mathrm{CH}_{3}$ rotations for the ester group in PMMA. As we remarked then, there are two effects on the motion when replacing $\mathrm{H}$ by $\mathrm{D}$. The hopping frequency shifts down by a factor of 2 .

It is hard to predict what the isotope effect on the tunneling regime will be. We have some doubts that a cascade of crossovers according to Moreno ${ }^{9,10}$ is the appropriate model to take multilevel tunneling into account. We rather believe that the various tunnel rates shown in Fig. 1(b) are just weighted by the Boltzmann population on the vibrational levels. It should be noted that all calculated tunneling rates are below the experimentally determined attempt frequency $1 / \tau_{\infty}$. It is, however, not clear by how much phonon assistance would shift these coherent frequencies. Anyhow, if multilevel tunneling would be effective at higher temperatures, the assumption of a Markovian process needed for the validity of Eq. (2) would be void. The spectral density at the Larmor frequency would have to be calculated from underdamped modes, phase fluctuations, and the lifetimes of the $\mathrm{CD}_{3}$ groups on the vibrational levels. It might be that the systematic deviation of the apparent mean $\tau_{c}$ from the Arrhenius behavior below $100 \mathrm{~K}$, shown in Fig. 5, is just a result of such mechanisms. This, however, remains pure speculation, as long as no proper theory for multilevel phonon assisted tunneling is available. The line shape transition, on the other hand, is expected to be less affected, in its discrimination between fast and slow exchange, by tunneling effects. It is therefore probably safe to assume that the plateau becoming visible at low temperature in Figs. 9 and 11 can be attributed to a tunneling process.

\section{DISCUSSION}

We are left with one other feature of the data not described by the models so far (except for some speculation), the apparent deviation from Arrhenius behavior at about 100 
$\mathrm{K}$ in Fig. 5. Disregarding quantum mechanical tunneling for the moment we are left to consider other possibilities.

The temperature dependence of the correlation time data presented in Fig. 5 could be described by two thermally activated mechanisms with different activation energies, with the total correlation time given by an expression of the form

$$
\left(\tau_{c}^{\mathrm{tot}}\right)^{-1}=\left(\tau_{c}^{(1)}\right)^{-1}+\left(\tau_{c}^{(2)}\right)^{-1} .
$$

However, as we know from the line shape transition analysis of the last section, for temperatures above $70 \mathrm{~K}$ we are in the fast motion regime, where the longest correlation time gives the shortest relaxation time. The above interpretation of the experimental inverse correlation times as the sum of the inverse correlation times of two independent processes Eq. (11), implies that what is observed is relaxation through the slower process, i.e., shortest correlation times. This is incompatible with the expectations for the fast motion regime.

The possibility of an alternative mechanism to the $120^{\circ}$ methyl group reorienting motion was carefully analyzed in Ref. 29, but none could be found which is compatible with accepted quadrupolar order relaxation theories and which at the same time explains the trend of the experimental autocorrelation times in Fig. 5. We are therefore left with three possibilities: (i) an artifact in the data analysis coming from the influence of the approaching slow motion regime, (ii) effects of spin-diffusion, and (iii) an asymmetric distribution of correlation times.

a. Influence of approaching the slow motion regime. Here we have to recall that there are two different clocks in the system defined by $2 \pi \nu_{L}$ and $2 \pi \nu_{Q}$. The first one being responsible for the $T_{1}$ minimum according to Eq. (2), the second for the line shape transition, respectively. It is known from other systems with a probability distribution of $\tau_{c}$ that Eq. (2) is valid also for $\tau_{c}>1 / \omega_{L}$. However, when the $\tau_{c} \mathrm{~s}$ of the slowest reorienting $\mathrm{CD}_{3}$ groups are reaching $\omega_{Q}^{-1}$ on lowering the temperature then second-order time dependent perturbation theory from which Eq. (2) is derived may be less and less able to make the correct predictions. With the parameters determined in the preceding section the minimum of the average $T_{1 Q}$ should occur at $84 \mathrm{~K}$, whereas the first signs of the line shape transition are observed just below 80 $\mathrm{K}$. This accounts maybe for the data points below $80 \mathrm{~K}$ but does not explain why the apparent $\tau_{c}$ starts to deviate from the Arrhenius behavior already at $125 \mathrm{~K}$.

b. Effects of spin diffusion. Mutual spin flips between spin pairs with the same resonance frequency (spatial spin diffusion) or with different resonance frequencies (spectral spin diffusion) might explain some of the features shown in Fig. 5. Since the spin diffusion rate is temperature independent it would limit the time for the complete decay of $M_{Q}(t)$ to a constant value. The longest time we have observed for the complete decay of $M_{Q}(t)$ at all temperatures is about 10 $\mathrm{s}$, see Fig. 3. A spin-diffusion time of this order would, however, affect only the decays for temperatures above $150 \mathrm{~K}$. This means that spin diffusion cannot be made responsible for the observed deviations of the apparent $\tau_{c}$ below $125 \mathrm{~K}$.

c. Asymmetric distribution of activation energies. In our analysis of the quadrupolar order decay curves we have assumed a log-Gaussian distribution of autocorrelation times
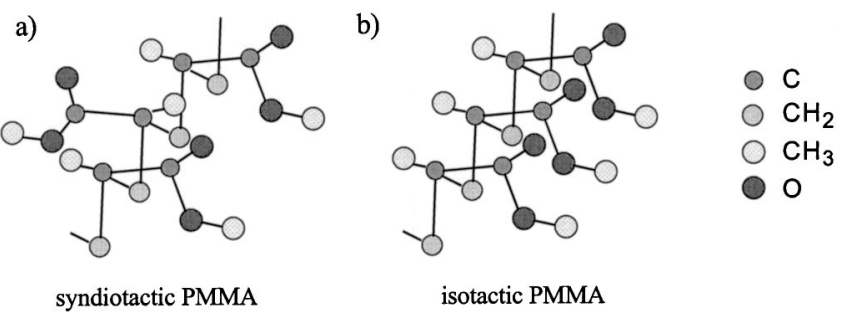

FIG. 10. Schematic conformations for stereospecific forms of PMMA. The thick lines represent the polymer chain backbone.

[see Eqs. (3) and (4)]. If we assume an asymmetric distribution of activation energies with a first moment shifted towards higher energies with respect to the actual position of the center of the Gaussian distribution on the activation energy axis, the result of the $\tau_{c}$ fit could be made to follow the high temperature Arrhenius law at least for the data points above $80 \mathrm{~K}$. Asymmetric distributions were already proposed in Refs. 22, 30, and 31. A detailed treatment is, however, beyond the scope of this work.

\section{EFFECT OF TACTICITY}

The relaxation behavior of the ester methyl group in PMMA has previously been extensively reported for the usually available predominantly syndiotactic samples. There is only one report in the literature of the effect of changing the sample structure to a predominantly isotactic structure (5) where it was reported that the rotational frequency is about $50 \%$ slower in the isotactic stereoisomer (at least for temperatures lower than about $250 \mathrm{~K}$ ). As can be seen from Fig. 10 , the syndiotactic sample alternates the position of the ester group on either side of the backbone while in isotactic sequences the ester groups are packed adjacently. One of the consequences of this different arrangement is that the glass transition temperature of the isotactic stereoisomer is about $67 \mathrm{~K}$ lower than that of the syndiotactic version $(311 \mathrm{~K}$ as opposed to $378 \mathrm{~K})$. In Fig. 11 we compare the results presented above from the sample in which $88 \%$ sequences are isotactic and the remaining ones atactic, with one previously studied with $50 \%$ syndiotactic sequences, $10 \%$ isotactic, and the rest atactic. Two effects might be expected in the deuteron NMR spectra. First the intramolecular organization is more uniform in the so-called isotactic sample, and this might be expected to result in a narrower spread of barrier heights. Second the rather close packing might give rise to a higher barrier to rotation.

a. Line shape transition. From DMR broadband spectra we observed that the transition to the slow motion regime in the isotactic sample takes place at temperatures well above those of the syndiotactic sample. The line shape transition already begins below $80 \mathrm{~K}$ in the isotactic sample, while it starts about $20 \mathrm{~K}$ lower in the syndiotactic one. In Fig. 11(a) the broad band DMR spectra of the syndiotactic and isotactic samples are shown at $T=32 \mathrm{~K}$. The two DMR spectra are normalized so that the integral below both curves is the same. The slow motion regime spectrum is clearly more pronounced in the isotactic sample. In Fig. 11(b) the results of the line shape transition analysis discussed in Sec. IV are 


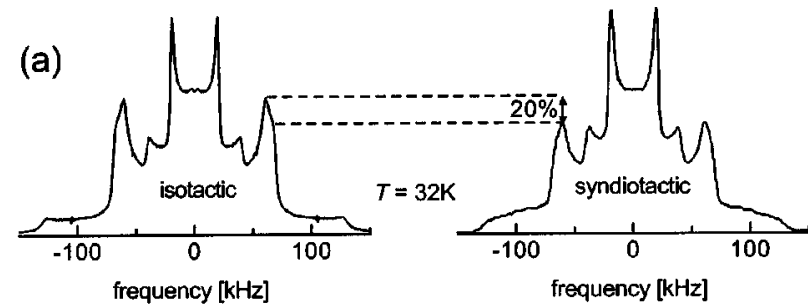

(b)

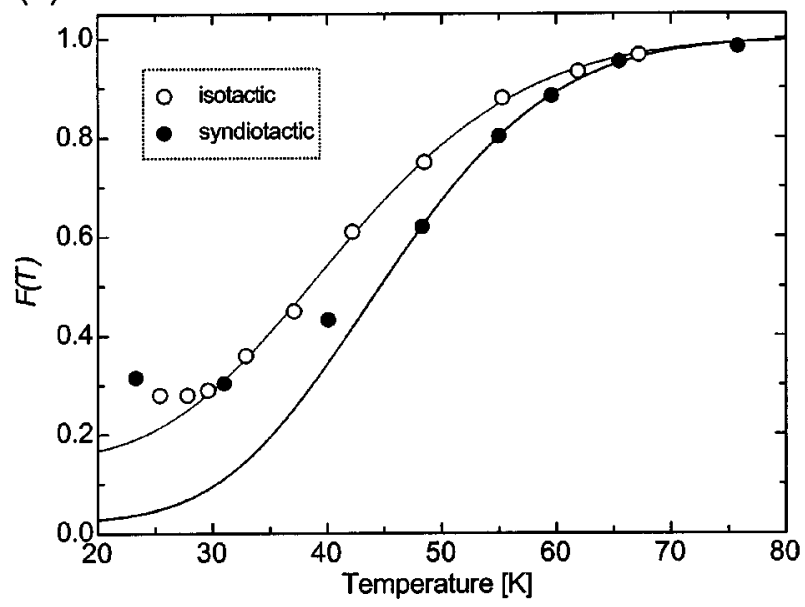

FIG. 11. Behavior of the syndiotactic and isotactic PMMA isomers in the slow motion regime. (a) Broad-band excited DMR spectra. (b) Line shape transition: experimental results (closed and open circles) and fit with Eq. 10 (solid line: isotactic PMMA, dashed line: syndiotactic PMMA).

reported together for both syndiotactic and isotactic samples. The comparison of the function $F(T)$ [Eq. (10)] for both polymers shows an earlier onset of the slow motion regime in the isotactic PMMA sample.

A temperature independent Gaussian distribution of activation energies is a good approximation for both isomers. We have reevaluated the line shape transition and the $T_{1 Q}$ data of Ref. 14 and used the improved two state model for the fit, see Fig. 11. A ratio $E_{a}^{s y n} / \sigma_{E_{a}}^{s y n}=2.85 \pm 0.05$ and $T^{c r}$ $=38.4 \pm 0.2 \mathrm{~K}$ were determined for the "syndiotactic" sample. Using the value $E_{a}^{s y n}=56 \pm 1 \mathrm{meV}(5.40 \mathrm{~kJ} / \mathrm{mol}) \mathrm{ob}-$ tained from $T_{1 Q}$ we get $\sigma_{E_{a}}^{s y n}=19.6 \pm 0.4 \mathrm{meV}(2.10 \mathrm{~kJ} / \mathrm{mol})$. These values have to be compared with $E_{a}^{i s o}=(62$ $\pm 1) \mathrm{meV}(5.98 \mathrm{~kJ} / \mathrm{mol})$ and $\sigma_{E_{a}}^{i s o}=(13.8 \pm 0.5) \mathrm{meV}(1.33$ $\mathrm{kJ} / \mathrm{mol})$. Similar differences in the activation energy distribution of mobile molecular units have been observed in other systems. ${ }^{18}$ Considering the Schrödinger equation for a threefold potential, one can easily calculate from the average activation energy $\left\langle E_{a}\right\rangle$ the methyl group potential barrier to rotation $V_{3}$ for both isomers: $V_{3}^{\text {syn }}=62 \mathrm{meV}(5.98 \mathrm{~kJ} / \mathrm{mol})$ and $V_{3}^{i s o}=69 \mathrm{meV}(6.66 \mathrm{~kJ} / \mathrm{mol})$. The small difference in $V_{3}$ explains why the variance of the Gaussian distribution of activation energies is broader for the so-called syndiotactic sample. This is so because this sample actually contains a fairly broad mixture of possible local sequences.

From the comparison of the results for syndiotactic vs isotactic PMMA we see that in isotactic PMMA the enhancement of the order on the polymer chain reflects itself principally on the line shape transition, i.e., (1) the line shape transition starts at higher temperature and (2) the low temperature DMR spectrum is more pronounced. Further, the higher local order of the isotactic sample in comparison with the local order of the syndiotactic one is seen in the relative width $\sigma_{E_{a}} / E_{a}$ of the corresponding distributions of $E_{a}$ which differ by a factor of 1.57 .

We can therefore say that to reach these results it is sufficient to compare the two polymers through the analysis of their line shape transition (Fig. 11). From the analysis of the correlation times no further information is gained, and the line shape transition is to be preferred as comparison method since it is far less time consuming and the evaluation procedure is much easier.

These results confirm and amplify the earlier neutron data showing that the ester methyl group in isotactic PMMA has a higher barrier to rotation and, at low temperatures, a lower frequency motion than the syndiotactic isomer. ${ }^{6}$ The implication is that these effects are caused by nonbonded interactions with neighboring groups.

\section{CONCLUSION}

The investigation of the dynamics of the deuterated ester methyl group by means of quadrupolar order spin-lattice relaxation measurements between $T=291 \mathrm{~K}$ and $T=70 \mathrm{~K}$ and of the deuteron line shape transition analysis between $T$ $=70 \mathrm{~K}$ and $T=23 \mathrm{~K}$ in an $88 \%$ isotactic sample of PMMA revealed a broad temperature dependent probability distribution of autocorrelation times $\left(\tau_{c}\right)$ for the $2 \pi / 3$ reorientation which can be explained by a Gaussian distribution of activation energies $\left[E_{a}=(62 \pm 1) \mathrm{meV}(5.98 \mathrm{~kJ} / \mathrm{mol}), \sigma_{E_{a}}=(13.8\right.$ $\pm 0.5) \mathrm{meV}(1.33 \mathrm{~kJ} / \mathrm{mol})]$.

We showed that the comparison between different stereospecific form of PMMA is easily obtained from their dynamic line shape transitions. Evidence for the higher local order and stronger nonbonded interactions with neighboring groups was found in the $88 \%$ isotactic sample with respect to a predominantly syndiotactic one $(50 \%$ syndiotactic, $30 \%$ atactic, and 20\% isotactic, Ref. 14).

We applied a log-Gaussian distribution of correlation times, corresponding to a Gaussian distribution of rotational barrier heights, to the analysis of the data because of a lack of any theoretical model for this distribution. On the other hand, we have no information concerning the real shape of the threefold potential, except for its periodicity. The shape could range from purely sinusoidal [as used in Fig. 1(b)] to, e.g., three intersecting parabolas with the corresponding shifts of the vibrational energy levels and tunnel frequencies, respectively. Provided that high temperature multilevel tunneling really takes place, one would have to question the meaning of the (in any case empirical) Arrhenius law as resulting from classical hopping over a hindering potential. Then the relation would very likely turn out to be the oversimplified result of a highly complex quantum mechanical phenomenon, where the Boltzmann population of the vibrational levels plays the essential role. 


\section{ACKNOWLEDGMENT}

This work was supported in part by the Swiss National Science Foundation.

${ }^{1}$ See, for example, Basic Features of the Glassy State, Proceedings of the Second International Workshop on non-crystalline Solids, San Sebastian, 1989, edited by J. Colmenero and A. Alegria (World Scientific, Singapore, 1989).

${ }^{2}$ J. Heijboer, J. M. A. Baas, B. van de Graaf, and M. A. Hoefnagel, Polymer 28, 509 (1987).

${ }^{3}$ J. G. Gomez Ribelles and R. Diaz Calleja, J. Polym. Sci., Polym. Phys. Ed. 23, 1297 (1985).

${ }^{4}$ N. G. McCrum, B. E. Read, and G. Williams, Anelastic and Dielectric Effects in Polymeric Solids (Wiley, New York, 1967).

${ }^{5}$ K. Schmidt-Rohr, A. S. Kulik, H. W. Beckham, A. Ohlemacher, U. Pawelzik, C. Boeffel, and H. W. Spiess, Macromolecules 27, 4733 (1994).

${ }^{6}$ B. Gabrys, J. S. Higgins, K. T. Ma, and J. E. Roots, Macromolecules 17, 560 (1984).

${ }^{7}$ G. Floudas and J. S. Higgins, Polymer 33, 4121 (1992).

${ }^{8}$ V. Arrighi, J. S. Higgins, A. N. Burgess, and W. S. Howells, Macromolecules 28, 2745 (1995).

${ }_{9}^{9}$ A. J. Moreno, A. Alegria, J. Colmenero, and B. Frick, Macromolecules 34, 4886 (2001).

${ }^{10}$ A. J. Moreno, A. Alegria, J. Colmenero, and B. Frick, Phys. Rev. B 59, 5983 (1999)

${ }^{11}$ J. G. Powles and P. Mansfield, Polymer 3, 336 (1962).

${ }^{12}$ Y. Tanabe, J. Hirose, K. Okano, and Y. Wada, Polym. J. (Singapore) 1, 107 (1970).

${ }^{13}$ D. Cavagnat, S. Clough, and F. O. Zelaya, J. Phys. C 18, 6457 (1985).
${ }^{14}$ Stephan Pfändler, Ph.D. Dissertation, ETH Zürich, 1995.

${ }^{15}$ C. P. Slichter, Principles of Magnetic Resonance, Third enlarged and updated edition (Springer, Berlin, 1990)

${ }^{16}$ A. Abragam, The Principles of Nuclear Magnetism (Clarendon, Oxford, 1961).

${ }^{17} \mathrm{H}$. W. Spiess, in Rotation of molecules and nuclear spin relaxation, NMR basic principles and progress, Vol. 15, edited by P. Diehl, E. Fluck, and R. Kosfeld (Springer, New York, 1978).

${ }^{18}$ R. Böhmer, G. Diezmann, G. Hinze, and E. Rössler, Prog. Nucl. Magn. Reson. Spectrosc. 39, 191 (2001).

${ }^{19} \mathrm{H}$. W. Spiess (private communication).

${ }^{20}$ S. Wimperis, J. Magn. Reson. (1969-1992) 86, 46 (1990).

${ }^{21}$ G. L. Hoatson, T. Y. Tse, and R. L. Vold, J. Magn. Reson. (1969-1992) 98, 342 (1992).

${ }^{22}$ E. Rössler, M. Taupitz, K. Börner, M. Schulz, and H. M. Vieth, J. Chem. Phys. 92, 5847 (1990).

${ }^{23}$ Z. T. Lalowicz, U. Werner, and W. Müller-Warmuth, Z. Naturforsch. 43a, 219 (1988).

${ }^{24}$ G. Diezemann, H. Sillescu, and D. van der Putten, Z. Phys. B: Condens. Matter 83, 245 (1991)

${ }^{25}$ A. J. Moreno, A. Alegria, J. Colmenero, and B. Frick, Phys. Rev. B 59, 5983 (1999).

${ }^{26}$ A. Heuer, Z. Phys. B: Condens. Matter 88, 39 (1992).

${ }^{27}$ J. Tang, A. Pines, and S. Emid, J. Chem. Phys. 73, 172 (1980).

${ }^{28}$ W. Press, Single Particle Rotations in Molecular Crystals (Springer, Berlin, 1981).

${ }^{29}$ P. M. Cereghetti, Ph.D. Dissertation, ETH Zürich, 2000.

${ }^{30}$ E. Rössler, M. Taupitz, and H. M. Vieth, J. Phys. Chem. 94, 6879 (1990).

${ }^{31}$ E. Rössler, K. Börner, M. Schulz, and M. Taupitz, J. Non-Cryst. Solids 131, 99 (1991). 